\title{
Erratum to: Innovative technology shows impact of glycaemic control on peripheral retinal vessels in adolescents with type 1 diabetes
}

\author{
Valli Velayutham $^{1,2}$ • Paul Z. Benitez-Aguirre ${ }^{1,2}$ - Maria E. Craig ${ }^{1,2,3}$ - Gerald Liew ${ }^{1,2}$. \\ Tien Y. Wong ${ }^{4,5}$ - Alicia J. Jenkins ${ }^{2} \cdot$ Kim C. Donaghue ${ }^{1,2}$
}

Published online: 1 October 2017

(C) Springer-Verlag GmbH Germany 2017

\section{Erratum to: Diabetologia}

DOI https://doi.org/10.1007/s00125-017-4375-3

Unfortunately, the affiliations given for Kim C. Donaghue and Alicia J. Jenkins were incorrect in this paper. The correct affiliations are listed below.

1. The Children's Hospital at Westmead, 212 Hawkesbury Road, Westmead, NSW 2145, Australia

2. University of Sydney, Sydney, NSW, Australia

3. School of Paediatrics, University of New South Wales, Sydney, NSW, Australia

4. Department of Ophthalmology, University of Melbourne and Centre for Eye Research, East Melbourne, VIC, Australia

5. Singapore Eye Research Institute, Singapore, Republic of Singapore

The online version of the original article can be found at https://oi.org/ $10.1007 / \mathrm{s} 00125-017-4375-3$

Kim C. Donaghue

kim.donaghue@health.nsw.gov.au

1 The Children's Hospital at Westmead, 212 Hawkesbury Road, Westmead, NSW 2145, Australia

2 University of Sydney, Sydney, NSW, Australia

3 School of Paediatrics, University of New South Wales, Sydney, NSW, Australia

4 Department of Ophthalmology, University of Melbourne and Centre for Eye Research, East Melbourne, VIC, Australia

5 Singapore Eye Research Institute, Singapore, Republic of Singapore 